\title{
Biologische Auswirkungen des Abwassers einer Ol-Raffinerie in einem Vorlandgebiet an der Nordsee
}

\author{
DiETRICH KöNIG \\ Landesamt für Wasserwirtschaft Scbleswig-Holstein, Kiel
}

\begin{abstract}
Biological effects of the waste water of an oil refinery in a silting area at the North Sea. The knowledge of the effects of oil refinery water wastes on the area of discharge is important for general ecological reasons, as well as for water resources policies and water laws. The amount of mineral oils and of phenols in the purified waste water is, in the present case, tolerable; but the content of these matters concentrates in the mud of the ditches; it exceeds the initial values about 100 times or more (in addition to the amount of oils and fats present in mud organisms). As a consequence, the composition of plant and animal communities of the mud area has changed: Cyanophyceae are now predominant and the usual representatives of the macrofauna have decreased in number or disappeared altogether, except Nereis diversicolor, a rather euryplastic species which occurs nearly everywhere. Further controls are necessary in order to ascertain whether these biological consequences of the wastes disposed remain restricted to the area of little ditches in the immediate neighbourhood of the outlets or whether they may affect also animals and plants of mud flat areas farther away.
\end{abstract}

\section{EINLEITUNG}

Die folgenden Darlegungen über die Auswirkungen des Abwassers einer O1Raffinerie, der Deutschen Erdöl AG (DEA), Werk Heide-Hemmingstedt, können nur Hinweise in Form einer vorläufigen Mitteilung sein. Bei näherer Beschäftigung mit diesen Auswirkungen erwies sich immer mehr die Schwierigkeit, Tatsachen der chemischen und biologischen Verhältnisse festzustellen und die Zusammenhänge und $\mathrm{Ab}$ hängigkeiten $\mathrm{zwischen} \mathrm{den} \mathrm{chemischen} \mathrm{und} \mathrm{biologischen} \mathrm{Elementen} \mathrm{zu} \mathrm{erkennen.}$

\section{DAS VERLANDUNGSGEBIET}

Das Vorland, die Außendeichswiese, in welche das DEA-Abwasser hineingeleitet wird, liegt in der Nordostecke der Meldorfer Bucht in Dithmarschen (Abb. 1). Es ist, wie an der Westküste üblich, zur Hauptsache eine Rotschwingelwiese (Festucetum rubrae litoralis) von etwa 0,80 bis $1,0 \mathrm{~m}$ über MThw Höhenlage; nach dem Watt zu geht es in ein Puccinellietum maritimae über; an das Watt grenzt es meist mit einer einige dcm hohen Stufe (Abb. 2-5). Das fein- bis mehlsandige Watt zum Hafenstrom 
hin ist teils ohne Bewuchs, teils ist es von relativ kleinflächigen Salicornia stricta-Beständen bewachsen, in welche Andelpolster (Puccinellia maritima) von der Vorlandkante her eindringen. Spartina ist in diesem Wattabschnitt noch kaum beteiligt; es feh-

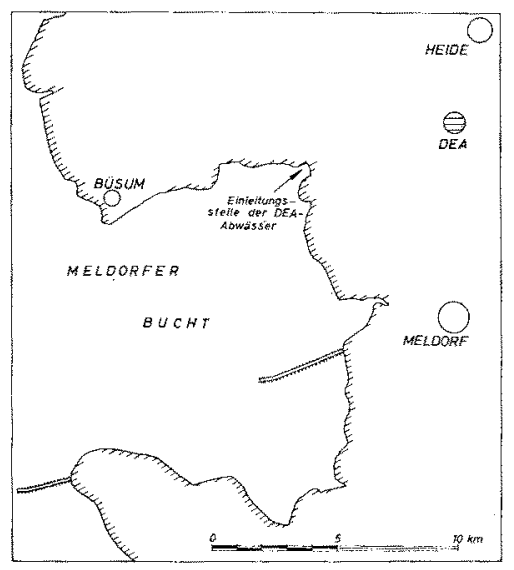

Abb. 1: Karte der Meldorfer Bucht in Dithmarschen (Schleswig-Holstein) mit Lage der Erdölraffinerie DEA und des Einleitungsgebietes der Raffinerie-Ábwässer

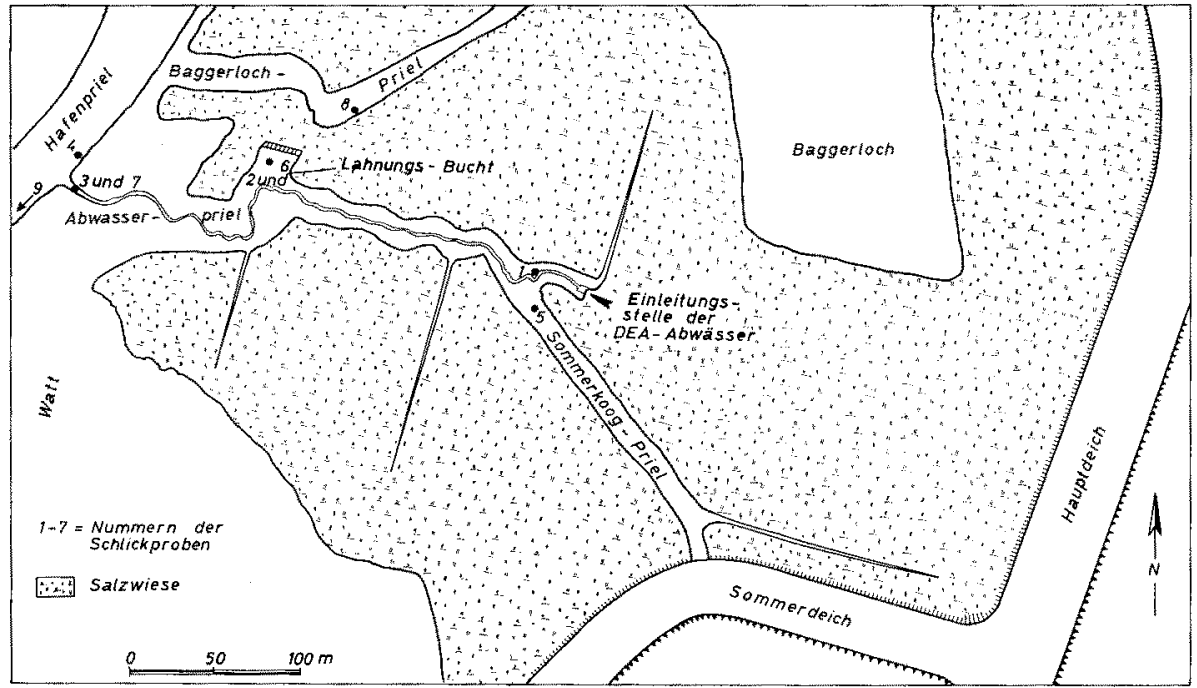

Abb. 2: Karte des Einleitungsgebietes

len auch Aster- und Suaeda-Bestände. Auf den wattnahen Vorsprüngen der Rotschwingelwiese sind qm-große flache Pfützen, welche meist Algenwatten enthalten.

Die Priele - Baggerlochpriel, Abwasserpriel und Sommerkoogpriel - sind mindestens $1 \mathrm{~m}$ unter die Wiesenfläche eingeschnitten. Der Hafenpriel reicht bis unter MTnw. So besteht im Abwasserpriel ein relativ starkes Gefälle von etwa $3 \mathrm{~m}$ auf 
$300 \mathrm{~m}$ Priellänge. Das Abwasser wird also bei Ebbe schnell in den Hafenpriel hinuntergeführt. Infolge des ständigen starken Wasserstromes hat sich hier am Prielgrunde eine schärfere Erosionsrinne eingeschnitten als an den benachbarten Prielen (Abb. 4, 5).

\section{BODENVERHALTNISSE UND BESIEDLUNG}

Der Boden des Vorlandes und des Watts besteht vorwiegend aus Mehl- und Feinsand der Korngrößengruppen von 0,02 bis $0,2 \mathrm{~mm}$. Von den Organismen sollen hier nur diejenigen kursorisch erwähnt werden, welche bei den vorliegenden überschlägigen

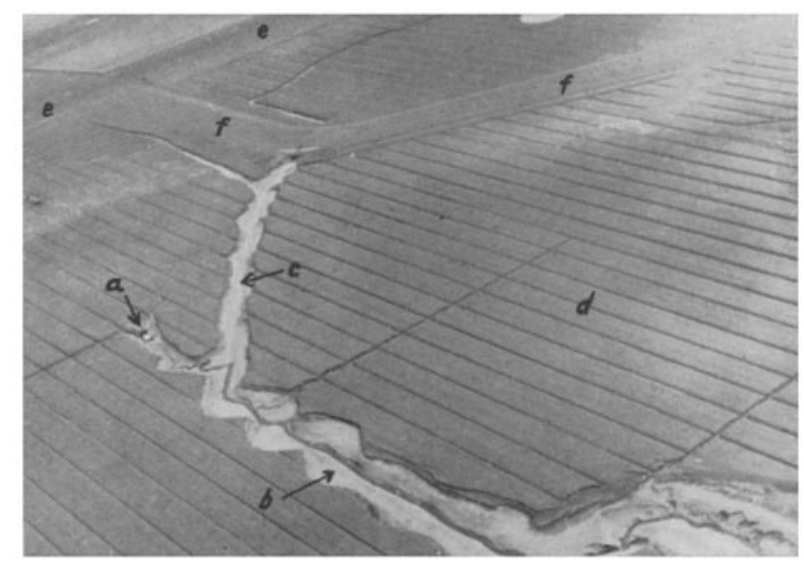

Abb. 3: Luftbild des Einleitungsbauwerkes (a), des Abwasserpriels (b), des Sommerkoogpriels (c), des Vorlandes (d), des Hauptdeiches (e) und des Sommerdeiches (f). Abstand der parallelen Gräben (Grüppen) auf dem Vorland $10 \mathrm{~m}$. (Aufnahme: D. KöNIG, 23. April 1965, Freigabe: SH - $168-138$ )

Erhebung vermerkt wurden. Im Sediment der Priele, soweit die Strömungsverhältnisse es zulassen, und auf den Wattflächen darüber leben von Haus aus von den Vertretern der Makrofauna vor allem: an Würmern Nereis diversicolor, Heteromastus filiformis, an Mollusken Macoma baltica, weniger Cardium, noch weniger Mya, viel Hydrobia ulvae, Alderia modesta auf Vaucheria-Polstern in den Vorlandgrüppen (Gräben), dort auch Assiminea grayana, an Krebsen Coropbium volutator, Carcinus, im Frühjahr zeitweise Eriocheir. Von größeren Algen herrschen Enteromorphen vor, wahrscheinlich vor allem E. linza und E. compressa. Auf dem Priel- und Wattsediment entwickelt sich eine ziemlich artenreiche Diatomeenflora. In den oberen kleinen Entwässerungsgrüppen des Vorlandes und stellenweise auf den obersten Partien des Watts kann es zu mehr oder weniger ausgedehnten Siedlungen von Vaucherien und Cyanophyceen, besonders Oscillatoria, kommen, doch treten diese flächenmäßig im Aspekt des gesamten Watt-Priel-Gebietes gegenüber den Diatomeen normalerweise weit zurück. 


\section{DIE ABWASSERVERHALTNISSE DER DEA}

In der Raffinerie der DEA von Heide-Hemmingstedt laufen stündlich bis etwa $200 \mathrm{~m}^{3}$ Abwasser ab. 1961 wurde eine 7,5 km lange Abwasserleitung vom Werk aus durch die Marsch und durch den Seedeich (= Hauptdeich = Landesschutzdeich) gelegt. Das Abwasser fließt seit Sommer 1962 hindurch.

Als Reinigungsverfahren wurde von der DEA nach Prüfung mehrerer Methoden das Biolator-Verfahren der Firma WABAG Wasserreinigungsbau Kulmbach gewählt. Es ist bei JACOBS (1965) beschrieben. Das Abwasser wird hierbei mechanisch und biologisch behandelt. Die Reinigungsanlage war in den ersten Jahren noch mit den üblichen Kinderkrankheiten behaftet. Dies braucht nicht verschwiegen zu werden, da

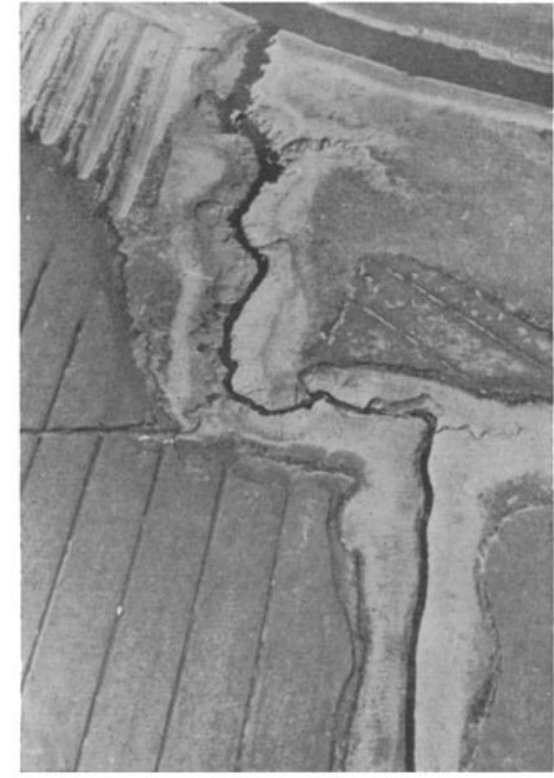

Abb. 4: Unterlauf des Abwasserpriels, der am oberen Bildrand in den Hafenpriel mündet. Bildmitte rechts die "Lahnungsbucht". Abwasserpriel mit scharf durch Erosion eingeschnittener Niedrigwasserrinne. (Aufnahme: D. KönıG, 23. April 1965, Freigabe: SH - $168-137)$

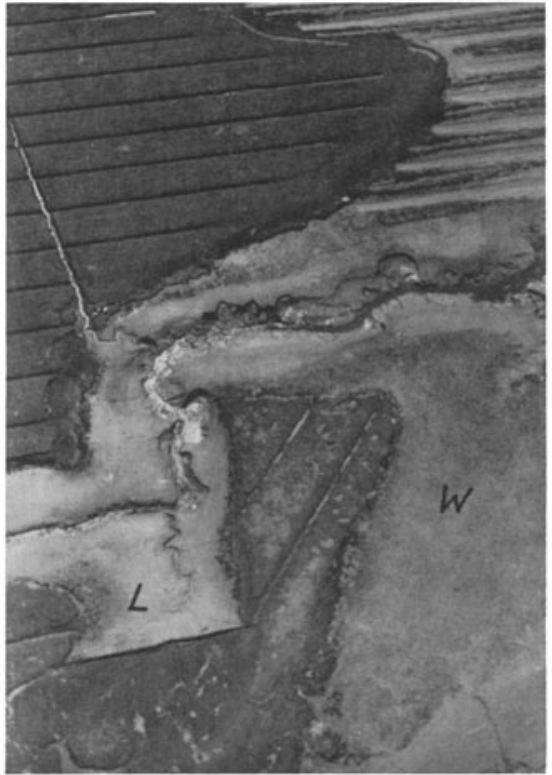

Abb. 5: Abwasserpriel, Fließrichtung von links nach rechts. $\mathrm{L}=$ Lahnungsbucht, $\mathrm{W}=$ Watt zwischen Abwasser- und Baggerlochpriel. (Aufnahme: D. KöNIG, 23. April 1965, Freigabe: SH - $168-134)$

erstens die Bemühungen der Werksleitung um eine wirklich intensive Abwasserreinigung offenkundig sind, und da zweitens die vorgekommenen Mißgeschicke in anderen Fällen als Lehre dienen können. Das ablaufende Abwasser war anfangs (JAcobs 1965) ziemlich verschiedenartig und sein Chemismus oft unbefriedigend $\left(\mathrm{NH}_{4}\right.$-Gehalt oft $>100 \mathrm{mg} / \mathrm{l}$, Phenole $>5 \mathrm{mg} / \mathrm{l}$ ). Das mußte in Kauf genommen werden angesichts der abwassertechnischen und -biologischen Schwierigkeiten; ist doch für die chemisch und 


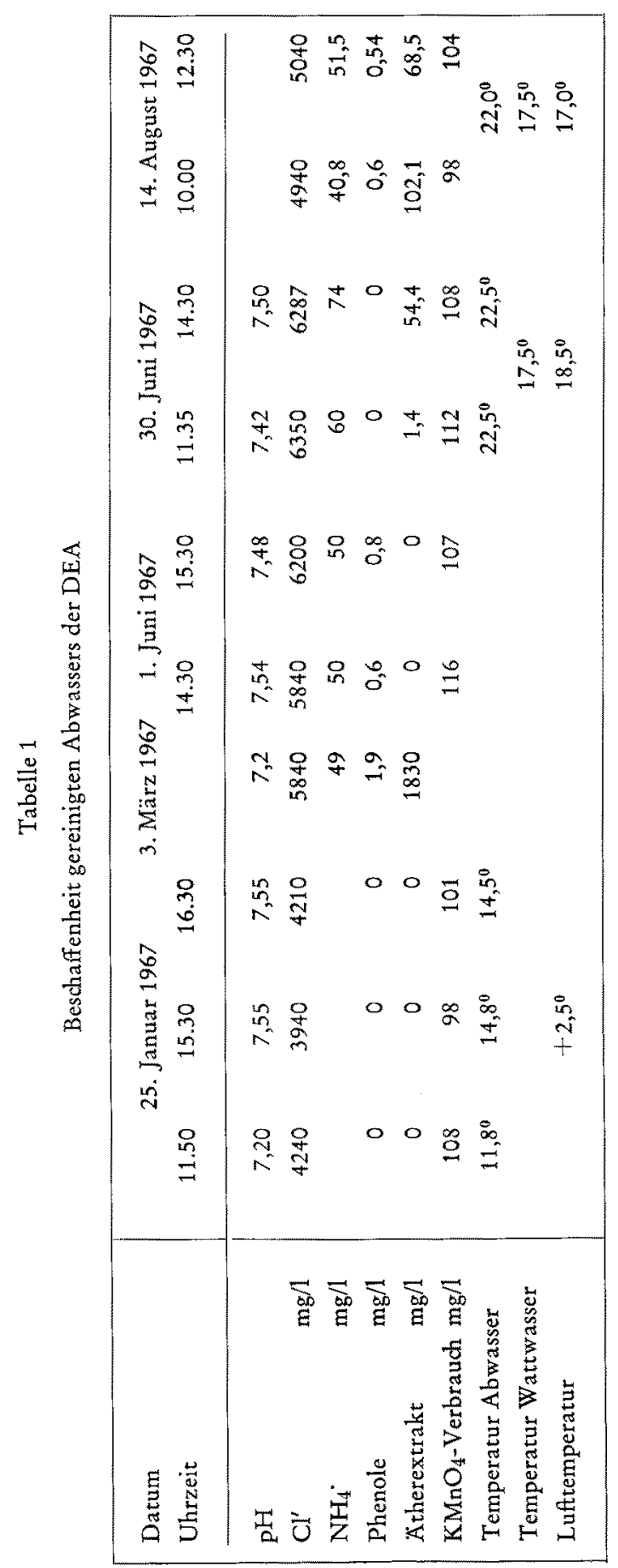


besonders biologisch zum Teil schwer angreifbaren und sehr verschiedenartigen Inhaltsstoffe solchen Raffinerie-Abwassers nicht einfach eins der für häusliche Abwässer anwendbaren Reinigungsverfahren ohne spezielle Anpassungen an diese Besonderheiten brauchbar.

Die Beschaffenheit des gereinigten Abwassers zur heutigen Zeit wird aus den in Tabelle 1 zusammengestellten Stichproben ersichtlich. Sie betreffen das nicht nur mechanisch-biologisch, sondern auch chemisch (mit Fällungsmitteln) behandelte Abwasser, während die bei JACOBS (1965) aufgeführten Werte aus der Zeit stammen, als wohl schon die biologische, aber noch nicht die chemische Abwasserbehandlung stattfand. Wie man sieht, liegen die Werte für ätherextrahierbare Stoffe und Gesamtphenole, wenn auch nicht immer, so doch wohl häufig im erträglichen Bereich. Als erträglich wird nach den derzeitigen Erfahrungen angesehen: petrolätherlösliche Stoffe bis $5 \mathrm{mg} / \mathrm{l}$, Gesamtphenole bis $2 \mathrm{mg} / \mathrm{l}$. Bemerkenswert ist die gegenüber der Umgebung ständig erhöhte Temperatur des Abwassers am Auslauf. Besonders im Winterhalbjahr ist das auffällig. Vermutlich wirkt sich dies aus auf die Stoffumsetzungen überhaupt, auf die Entwicklung der Kleinalgen etc.

\section{AUSWIRKUNGEN DES ABWASSERS}

Trotz offensichtlicher Sorgfalt beim Ausbau und bei der Wartung der Kläranlage traten und treten doch vermutlich auch neuerdings öfter kleinere oder größere Pannen auf, welche eine Belastung des Vorfluters bedeuten.

Eine ungewöhnliche Verschmutzung geschah gleich im ersten Jahr, kurz nach Inbetriebnahme, im September 1962. Sie soll hier geschildert werden, da sie anscheinend dem Abwasserpriel schon seinen endgültigen Zustand gab. Im Werk brach ein Damm eines Erdbeckens für den Ollschlamm, und eine große Menge dieses Schlammes wurde mit in die Abwasserleitung und in das Vorlandgebiet gepumpt. An dem Tage war gerade auch infolge starken Windes übernormal hohes Wasser, welches das Vorland ïberflutete. So floß der schwarze Olschlamm großenteils nicht im Abwasserpriel in das Wattenmeer, sondern er wurde durch das steigende Flutwasser auf etwa 12 ha des Vorlandes ausgebreitet und lagerte sich mit dem Sinken des Wassers auf dem Rasen ab, vor allem in der Nähe der zahlreichen Entwässerungsgrüppen. Das bedeutete einen beträchtlichen Schaden wegen des Ausfalls der betreffenden Flächen als Schafweide. Die stärkste Ablagerung wurde durch eine von der DEA beorderte Arbeitskolonne von $H$ and abgehoben und vergraben. Die Olschmiere war im gesamten Abwasserpriel bis zu dessen Mündung in den Hafenpriel vorhanden. Im Hafenpriel selbst waren dennoch keine Ablagerungen zu bemerken. Auf die Wattorganismen hatte der Olschlamm eine verheerende Wirkung. Das war am auffälligsten an den toten Würmern Nereis diversicolor. Diese Art besiedelt dieses Schlickprielwatt in üblicher Menge. Sie ist bekanntlich eine der gegen Schwankungen der verschiedenen Biotopfaktoren, auch der chemischen, am wenigsten empfindlichen Arten. So lebt sie in $\mathrm{O}_{2}$-armem und $\mathrm{H}_{2} \mathrm{~S}$ haltigem Boden, wenn sie dort ihre mit der Außenluft in Verbindung stehenden Gänge anlegen kann. Im besprochenen Fall lag am 20. September, also am Tage nach dem Ausbruch des Olschlamms, der gesamte Nereis-Bestand im Watt der "Lahnungsbucht" 
tot an der Oberfläche (Abb. 2). Die Tiere hatten offenbar versucht, den in die Gänge eindringenden chemischen Stoffen zu entkommen und waren ins Freie gekrochen, dann aber an Ort und Stelle an Vergifung gestorben. Auch im Abwasser-Priel und an ins Wasser ragenden Gegenständen hingen tote Nereis. Im Laufe des Winters und Frühjahrs verschwanden die offen sichtbaren Spuren dieses Zwischenfalls auf dem Rasen durch Vergehen des alten und Nachwachsen des neuen Grases. Wie der Olschlamm im Schlick des Priels, wo sich natürlich auch solcher in Massen abgelagert hatte, abgebaut wurde, konnte nicht genauer verfolgt werden.

Immerhin war die Abwasserreinigung doch im allgemeinen in den ersten Jahren schon so intensiv, daß z. B. nicht nur Cyanophyceen (vorwiegend Oscillatorien) in Massensiedlungen, sondern auch Nereis und stellenweise Macoma-Brut, Capitelliden, Hydrobia-Brut im Priel oder daneben auf dem höheren Watt neben Salicornia-Vorposten zu leben vermochten.

Am 25. Januar 1967 wurde beobachtet, daß ein Olfilm, der aus dem Abwasserrohr herauslief, bei Hochwasser auf dem Prielwasser schwamm, an den Rand gedrängt wurde und sich bei Ebbe auf der trockengefallenen Diatomeen-Haut am Prielrande absetzte. Am 19. August 1963 kamen Olschlieren, die mit dem Prielwasser bei Ebbe in den Hafenpriel geflossen waren, mit dem Flutstrom in den Abwasserpriel zurück; sie konnten daher eventuell bei der nächsten Ebbe doch noch im Bereich des Abwasseroder des Sommerkoog-Priels oder der mit ihnen verbundenen Grüppen abgelagert werden. Am 14. August 1967 schwammen im Sommerkoogpriel mit dem ablaufenden Wasser ständig außer Cyanophyceen-Fladen auch Olschlieren abwärts, dann und wann quollen Olblasen aus dem Schlick an die Wasseroberfläche. Offenbar haben sich oftmals derartige Olfilme, eventuell vermischt mit anderen chemischen Stoffen, dem Schlick verbunden, wobei wahrscheinlich die Cyanophyceen, vielleicht zum Teil auch Diatomeen als Auffanglager oder als physiologische Speicher wirken.

Die biologischen und die chemisch-physikalischen Ursachen der Veränderung im Bereich des Abwasserpriels können nicht scharf voneinander getrennt werden; sie greifen offenbar sehr ineinander, müssen daher auch in der Darstellung neben- und miteinander gebracht werden.

Entnimmt man an beliebiger Stelle des Abwasserpriels, des Sommerkoogpriels oder anderer benachbarter Gräben, welche mit ihm kommunizieren, Proben des schwarzen Schlicks, dann fällt ein meist sehr intensiver „Raffinerie“-Geruch auf, welcher auch über dem ganzen Gelände liegt und bis zum Deich bemerkbar ist. Die Befunde an einigen derartigen Beispielsproben sind in Tabelle 2 dargestellt (Lage der Entnahmepunkte siehe Abb. 2). Es handelt sich bei Nr. 1, 3, 5 und 7 um Abwasserschlick, bei den übrigen zum Vergleich um wenig oder nicht verschmutzten Schlick, bei Nr. 2 und 6 aus der Lahnungsbucht, bei Nr. 4 und 9 vom Hafenpriel, bei Nr. 8 vom Baggerlochpriel.

Die Werte für petrolätherextrahierbare Stoffe und Gesamtphenole sind auch in den unverschmutzten Prielen recht hoch. Nach dem Geruch zu urteilen, können auch in diesen Proben Stoffe aus dem Mineralölwerk beteiligt sein, doch sind in den WattprielSedimenten stets auch pflanzliche und tierische Organismen vorhanden, welche Ferte und Öle in ihren Körpern enthalten. Besonders Diatomeen mit bekanntlich hohem O1gehalt sind hier wesentlich beteiligt. Der Anteil aus diesen Organismen an Fettstoffen ist natürlich im Gesamtwert der Schlickprobe enthalten. Sein genauer Wert ist aber 
Tabelle 2

Untersuchung von Schlickproben aus Abwasserprielen. Chemische Analysen: Städtisches Laboratorium Kiel

\begin{tabular}{|ccccc|}
\hline Datum & $\begin{array}{c}\text { Station- } \\
\text { Nr. }\end{array}$ & $\begin{array}{c}\text { Sediment- } \\
\text { Tiefe } \\
(\mathrm{cm})\end{array}$ & $\begin{array}{c}\text { Petrol- } \\
\text { ätherextrahierbare } \\
\text { Stoffe } \\
\text { (mg/l } \\
\text { Sediment-Wasser) }\end{array}$ & $\begin{array}{c}\text { Phenole } \\
\text { (mg/l } \\
\text { Sediment-Wasser) }\end{array}$ \\
\hline 30. Juni & $1 \mathrm{a}$ & $0-1$ & 10932 & 53,0 \\
1967 & $1 \mathrm{~b}$ & $5-10$ & 6674 & 46,5 \\
& $2 \mathrm{a}$ & $0-1$ & 6985 & 46,8 \\
& $2 \mathrm{~b}$ & $5-10$ & 1569 & 48,8 \\
& 3 & $1-2$ & 4705 & 46,1 \\
14. August & $5 \mathrm{a}$ & $1-2$ & 7663 & 11,0 \\
1967 & $0-1$ & 5038 & 8,8 \\
& $5 \mathrm{~b}$ & 5 & 5696 & 11,6 \\
& 6 & $1-2$ & 2250 & 5,3 \\
& $7 \mathrm{a}$ & $0-2$ & 8156 & 6,3 \\
& $7 \mathrm{~b}$ & 5 & 3394 & 11,4 \\
& 8 & $0-2$ & 1632 & \\
\hline
\end{tabular}

kaum festzustellen, da er von der Menge und Art der beteiligten Organismen abhängt und dementsprechend stark schwanken kann. Immerhin ist aus den Zahlen der Tabelle 2 doch ein Unterschied $z$ wischen verschmutztem und unverschmutztem Prielschlick im Ölgehalt ersichtlich. Diesem Problem wird weiter nachgegangen, auch in $\mathrm{Zu}$ sammenarbeit mit dem chemischen Laboratorium der DEA. Bei Vergleichsproben aus unverschmutzten Gewässern aus anderen Gebieten traten überraschenderweise ebenfalls hohe Phenolwerte auf, welche den Verdacht aufkommen lassen, daß bei der Phenolbestimmung nach den Deutschen Einheitsverfahren auch andere Stoffe als Phenole in derselben Weise wie diese reagieren. Auch diese Erscheinung erfordert weitere Untersuchungen. Man wird zur Zeit wenigstens sagen können, daß die Phenol-Bestimmungen im freien abfließenden Wasser tatsächlich die zu untersuchenden chemischen Gruppen betreffen, daß dagegen im feuchten Sediment auch andere Anteile ähnlich wirken können.

Ober Aussehen und Struktur des Schlicks im abwasserbelasteten Priel im Vergleich mit normalem Prielschlick der Umgebung ist zu sagen: $\mathrm{Da}$ sich solche Dinge schwer durch chemische und physikalische Methoden exakt erfassen lassen - auch der LiNKEsche Bodenhärtemesser (LiNke 1939) ließ sich hier nicht recht als Maßstab verwenden wurden wenigstens Farbfotos als Belege angefertigt. Der Schlick ist tiefschwarz von Eisensulfid, viel dunkler als der Schlick des benachbarten unverschmutzten Gebietes, welcher natürlicherweise ebenfalls Eisensulfid-Verdunklung aufweist. Die Oxydationsschicht ist nur Bruchteile von Millimetern dick (normalerweise meist wenigstens einige Millimeter). Am Grunde des Priels ist der Schlick oberflächlich mehr suppig-weichflüssig als sonst, oft mit weißlicher Beimischung, offenbar durch amorphen Schwefel. Andererseits wird er weiter oben im Prielquerschnitt, nahe der Hochwassergrenze, wo die Überflutung nur kurzfristig geschieht und manchmal ganz ausbleibt, viel trockener 
und härter, als es an diesen Vorlandprielen üblich ist. Das kommt wohl vor allem durch die Entwicklung von Blaualgenhäuten auf seiner Oberfläche. Jedoch treten diese Cyanophyceen-Siedlungen in wechselnder Intensität auf, je nach den Wasserverhältnissen durch Überflutung oder Niederschläge. Sie neigen offenbar dazu, eben wegen der zeitweisen besonderen Härte und Trockenheit des Prielsediments, in trockenen Zeiten zu verkümmern. So war es im Juni 1967. Am 1. Juni 1967 war die CyanophyceenDecke sehr stark ausgebildet. Im letzten Maidrittel mit $31 \mathrm{~mm}$ Niederschlag war das Substrat genügend feucht gehalten. Im Juni 1967 dagegen fielen insgesamt nur $16 \mathrm{~mm}$ Niederschlag. Deshalb war am 30. Juni 1967 der Schlick im oberen Teil der Böschung des Abwasserpriels besonders hart und trocken. Der Wind war in diesem Monat so schwach gewesen, daß es zu keiner Uberflutung des Vorlandes und damit länger andauernder Durchtränkung des Prielschlicks kam. Cyanophyceen-Siedlungen waren daher nur in Resten sichtbar, sei es, daß sie eingetrocknet und abgeblättert, abgespült oder abgeweht worden waren. Auch der Juli 1967 war im ganzen zu trocken, doch gab es in diesem Monat wie auch im August manche gewittrigen Störungen mit böigen Winden. Diese wirkten sich in dem hier betrachteten Prielsystem derart aus, daß im Abwasserpriel eine kräftige rückschreitende Erosion und stellenweise erheblicher $\mathrm{Ab}$ bruch an den Böschungen des mäandernden Gerinnes erfolgte. Beides geschah infolge der unnormalen Strukturverhältnisse des Sediments in beträchtlich schrofferen Formen, mit höheren Erosionsstufen (senkrechter Abbruch 30 bis $50 \mathrm{~cm}$ und mehr), als es an den benachbarten Prielen mit dem normalen, plastischen Sediment der Fall war.

\section{AUSWIRKUNG AUF DIE BIOZONOSEN}

Die Auswirkung auf die Biozönosen kann hier nicht in allen Einzelheiten geschildert werden, da sie vor allem noch nicht ganz bekanntgeworden ist. Sie soll weiter verfolgt werden. Doch können immerhin einige Hinweise gegeben werden, welche durch photographische Belege verdeutlicht werden.

Am auffälligsten ist beim Oberblick über die abwasserbelasteten Priele und Gräben das starke Vorherrschen ron Cyanophyceen-Rasen, welches schon oben erwähnt wurde. Vor allem sind Oscillatoria-Arten mengenmäßig dabei beteiligt. Die taxonomischen Einzelheiten werden später geklärt werden können, da Herr Dr. SCHwABE (Max-Planck-Institut für Limnologie, Plön) sich dankenswerterweise bereit erklärt hat, sich in gemeinsamer Arbeit mit ihnen zu befassen.

Diatomeen, welche normalerweise die Oberfläche des Prielschlicks besiedeln, treten für das bloße Auge wegen der Cyanophyceen-Rasen zurück, sind aber lebend vorhanden. Ein Uberblick über die gehäuft hier vorkommenden Arten ist in Tabelle 3 gegeben.

Es sind hier von den viel zahlreicheren überhaupt beobachteten Benthos-Arten nur die häufigen zusammengestellt, welche in den frischen Proben lebend gesehen wurden oder infolge ihrer Häufigkeit für hier lebend gehalten werden können. Die Proben sind in der Tabelle von links nach rechts nach abnehmender Einwirkung von Raffinerie-Abwasser angeordnet (vgl. Karte, Abb. 2). Probe (a) stammt von nahezu der gleichen Stelle an verschiedenen Tagen; einige Proben aus Cyanophyceen-Siedlungen; 


\section{Tabelle 3}

Häufigste Arten an Bodendiatomeen in der Umgebung des Abwassereinlaufes der DEA.

(CC = massenhaft, $\mathrm{C}=$ sehr häufig, $+\stackrel{=}{=}$ in beträchtlicher Zahl)

Beschreibung der einzelnen Entnahmepunkte: (a) Abwasserpriel neben dem DEA-Auslauf; 1. Juni trockene Oscillatoria-Haut; 30. Juni schwefelweiße Steine und Klei; 14. August Cyanophyceen-Rasen; (b) kleiner Nebengraben des Abwasserpriels, feuchte CyanophyceenDecke; (c) kleines Rinnsal in Nebengraben des Abwasserpriels, schwimmende fädige Diatomeen-Flocken; (d) Abwasserpriel bei der Einmündung des Sommerkoog-Priels, abblätternde Cyanophyceen-Haut; (e) Abwasserpriel $10 \mathrm{~m}$ oberhalb der Mündung in den Hafenpriel; (f) Sommerkoog-Priel, schwimmende Cyanophyceen-Fladen; (g) Sommerkoog-Priel, $10 \mathrm{~m}$ oberhalb Zusammenfluß mit dem Abwasserpriel, gewachsene Cyanophyceen-Siedlung; (h) Lahnungsbucht, Diatomeen-Flecken zwischen Puccinellia maritima-Jungpflanzen; (i) Lahnungs-

\begin{tabular}{|c|c|c|c|c|c|c|c|c|c|c|}
\hline \multirow{2}{*}{ Species } & \multirow[b]{2}{*}{$\begin{array}{r}\text { Stationen } \\
\text { Datum (1967) }\end{array}$} & \multicolumn{9}{|c|}{$\begin{array}{l}\text { Einwirkung von Raffinerieabwasser: } \\
\text { stark }\end{array}$} \\
\hline & & 1.6. & 30.6 . & 14.8. & $\begin{array}{c}\mathrm{b} \\
1.6 .\end{array}$ & $\begin{array}{c}\mathrm{c} \\
1.6 .\end{array}$ & $\frac{\mathrm{d}}{30.6}$ & $\begin{array}{c}\mathrm{e} \\
14.8 .\end{array}$ & $\stackrel{f}{f} .8$. & $\stackrel{\mathrm{g}}{14.8 .}$ \\
\hline Amphiprora & alata Kürz. & & & & & & & & & \\
\hline Amphora & $\begin{array}{l}\text { coffeaeformis AGARDH } \\
\text { exigua GREG. } \\
\text { lineolata EHR. } \\
\text { proteus GREG. }\end{array}$ & & $\mathrm{C}$ & & $\mathrm{C}$ & $\underset{\mathrm{C}}{\mathrm{CC}}$ & $\begin{array}{l}+ \\
+\end{array}$ & & $\begin{array}{l}\mathrm{CC} \\
+\end{array}$ & $\begin{array}{l}\mathrm{C} \\
+\end{array}$ \\
\hline Caloneis & formosa (GREG.) CL. & & & & & & & & & \\
\hline Gyrosigma & fasciola (EHR.) CL. & & & & & & & + & & \\
\hline Navicula & $\begin{array}{l}\text { bahusiensis GRUN. } \\
\text { consentanea Hust. } \\
\text { cryptocepbala KüTz. } \\
\text { digitoradiata (GREG.) A. ScH. } \\
\text { forcipata GREv. } \\
\text { gregaria DoNK. } \\
\text { budsonis GRUN. } \\
\text { pavillardi HUST. } \\
\text { rostellata KüTz. } \\
\text { salinarum GRUN. }\end{array}$ & + & & $+/ \mathrm{C}$ & $+/ \mathrm{C}$ & C & $\begin{array}{l}+ \\
+/ C \\
+\end{array}$ & $\mathrm{CC}$ & $\begin{array}{l}\mathrm{C} \\
+/ \mathrm{C} \\
+ \\
+\end{array}$ & $\begin{array}{l}\mathrm{C} \\
+ \\
+ \\
+\end{array}$ \\
\hline Nitzschia & $\begin{array}{l}\text { acuminata (W. SM.) Grun. } \\
\text { constricta (GREG.) Grun. } \\
\text { cf. laevis Hust. } \\
\text { cf. palea (KÜTz.) W. SM. } \\
\text { sigma (KüTz.) W. SM. } \\
\text { spec. }\end{array}$ & $\mathrm{CC}$ & & & $\begin{array}{l}\mathrm{C} \\
+\end{array}$ & $\mathrm{CC}$ & $\begin{array}{l}\mathrm{CC} \\
+\end{array}$ & & + & \\
\hline Pleurosigma & angulata v. quadr. (W. Sм.) v. Нск. & & & & & & & & & \\
\hline Stauroneis & gregorii RALFS & & & & & & & + & & \\
\hline Surirella & ovalis BRÉr. & & & & & & & + & & \\
\hline
\end{tabular}

Proben (h) bis (k) aus der Lahnungsbucht an zwei verschiedenen Tagen, jeweils in Abständen von einigen Metern voneinander, bei unterschiedlicher oberflächlich sichtbarer Besiedlung; Proben ( $m$ ) bis (o) von ganz ähnlichen benachbarten Stellen und vom selben Tage; Probe $(p)$ und $(q)$ vom selben Standort an einem anderen Tage; Probe ( $r$ ) bis $(t)$ vom Schlick des Baggerlochpriels, welcher kaum yom Raffinerie-Abwasser beeinflußt wird. 
Tabelle 3

bucht, nackter trockener Schlick; (j) Lahnungsbucht, gelblicher Diatomeen-Belag; (k) Lahnungsbucht, Cyanophyceen-Siedlung; (1) Hafenpriel, etwa $8 \mathrm{~m}$ oberhalb Mündung des Abwasserpriels, goldbrauner Diatomeen-Fleck; $(\mathrm{m})$ Watt zwischen Abwasser- und Baggerlochpriel, Diatomeen-Flecken in lockerem Salicomia-Puccinellia-(Spartina-)Bestand; (n) ähnlich (m); (o) ähnlich (m); (p) dasselbe Watt wie (m) mit Tadorna-Fraßspuren im Diatomeen-Belag; (q) ähnlich (p); (r) Baggerlochpriel, weicher Schlick, goldbrauner Anflug nahe Hochwassergrenze; (s) Baggerloch-Priel, grün-brauner Oberflächenbelag; (t) Baggerloch-Priel, gold-brauner Diatomeen-Belag auf Schlick; (u) unverschmutzte Vorland-Grüppe (kleiner Graben), Diatomeen-Watte

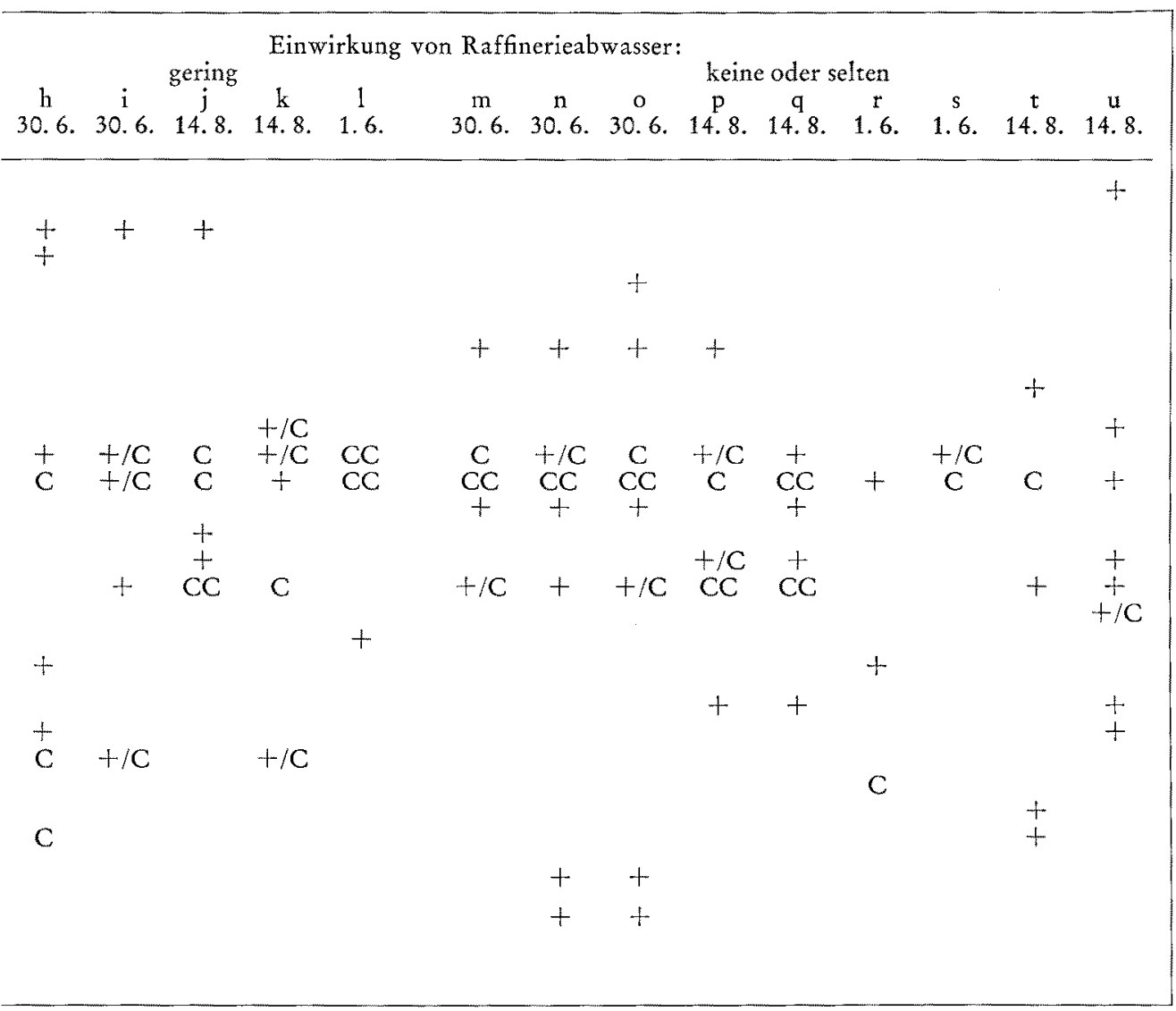

Die Tabelle zeigt folgendes: Nur wenige Diatomeen kommen in der unmittelbaren Nähe (2 bis $3 \mathrm{~m}$ ) des DEA-Auslaufs vor, darunter eine Nitzschia - Nitzschia palea ist bekannt für Vorkommen in Abwasserbereichen -, Amphora coffeaeformis (welche ich auch sonst oft in faulschlammigen Kleingewässern an der Küste fand) und Navicula consentanea, eine auf dem Watt häufige Art. Im übrigen sagen die Diatomeen-Proben jedoch nichts Genaues über Wirkungen der Abwasser auf die Lebensmöglichkeiten 
dieser Algen. Daß nur wenige Arten in jeder Probe massiert auftreten, ist nichts Ungewöhnliches; ähnlich findet man es sehr häufig im Watt und auch an anderen Standorten. Auch eine Verteilung nach den Verschmutzungsgraden ist nicht beweisbar. Die häufigsten Arten, Navicula cryptocephala und $N$. consentanea, auch $N$. budsonis, sind über die ganze Breite der Tabelle vorhanden, die übrigen mehr oder weniger wahllos verteilt. Es ist keinesfalls so, daß Amphora coffeaeformis auf chemisch extreme Standorte eingestellt wäre, sie kommt anderswo auch auf „weniger schwierigen“ Standorten vor. Am ehesten ist - auch nach sonstigen Beobachtungen am Watt - zu sagen, daß Caloneis formosa nicht auf den stark durch Raffinerie-Abwasser belasteten Stellen gedeihen kann; vielleicht ist das auch bei Navicula digitoradiata der Fall, welche auf sauerstoff reichen Standorten ohne starke Fäulnisprozesse oft sehr häufig ist.

Floristisch ist noch auf einige der aufgeführten Arten hinzuweisen, welche bisher von der schleswig-holsteinischen Westküste wenig bekanntgemacht worden sind. Navicula budsonis, welche durch HustedT (1955) wieder mehr ins Blickfeld der Diatomeenkundler gerückt worden ist, ist nach meinen Beobachtungen im Wattengebiet sehr häufig. Sie ist wohl meist mit $N$. pygmaea verwechselt worden. Im einzelnen kann hierauf an dieser Stelle nicht eingegangen werden. Bemerkenswert ist das gehäufte Auftreten von Navicula bahusiensis (der Name ist nach Hustedt 1962 genommen; Synonyme sind $N$. bremeyeri var. rostrata, HustedT 1939, und N. koenigi, BrockManN 1950) und von Navicula pavillardi. Beide Arten scheinen Standorte brackigen Charakters zu bevorzugen, wobei Einzelheiten der chemischen Erfordernisse noch unbekannt sind.

Ciliaten, Kleinwürmer und Kleinkrebse wurden nicht untersucht.

Bei der Makrofauna ist eine Verarmung der Artenzahl auffällig. Von den oben aufgeführten Arten, welche hierher gehören, ist im Abwasserpriel praktisch nur noch Nereis diversicolor übrig. Diese Art hat die Olschlamm-Katastrophe von 1962 natürlich längst überwunden. Sie fehlt in der am stärksten belasteten Stelle unmittelbar beim DEA-Auslauf, tritt aber weiter abwärts im Abwasserpriel schon in solcher Dichte auf, daß man beispielsweise am 14. August 1967 im unteren Prielabschnitt deutlich das typische knisternde Wattgeräusch von diesen Würmern hörte. Auf den höheren Wattflächen neben dem Abwasserpriel sind dann wenigstens zeitweise auch Macoma- und Hydrobia-Brut sowie Capitelliden neben Nereis vorhanden. Andere hierher gehörige Arten, wie Corophium volutator und Cardium, wurden dagegen in der Beobachtungszeit nicht gesehen.

So hat, insgesamt gesehen, eine deutliche Umstellung der Priel-Biozönosen stattgefunden, welche entsprechend den ökologischen Regeln bei einer Reihe von Gruppen anscheinend zu einer Artenverarmung unter diesen extremen Verhältnissen geführt hat, dabei aber nicht zu einer Dichtezunahme der übriggebliebenen Arten. Man kann also durchaus von einer Verarmung sprechen.

Im Hafenpriel wurden bisher keine gravierenden Anzeichen von Verschmutzung durch die Raffinerie-Abwässer gefunden. Deren Auswirkungen sind also zur Zeit auf das Gebiet des Abwasserpriels und der mit ihm kommunizierenden Gräben im Vorland beschränkt.

Es ist aber notwendig, sich folgendes klarzumachen. Bei Annahme von $4000 \mathrm{~m}^{3 /}$ Tag Abwasser und einem Olgehalt von $5 \mathrm{mg} / 1$ fallen am Tag $20000 \mathrm{~g}$, im Jahr 
(350 Tage) 7 t Ol aus dem Ablauf an. Die Hoffnung, daß dieses Oll ständig abgebaut wird, ist nach den zur Zeit vorliegenden Feststellungen nicht berechtigt. Deshalb erfordert dieses Problem weitere Aufmerksamkeit der Behörden bei der Kontrolle wie der Werksleitung beim Betrieb der Kläranlage. Dabei wären auch bakteriologische Untersuchungen wichtig zur Feststellung, ob Bakterien wie in der Biolator-Anlage auch im Freien beim Abbau die wesentliche Rolle spielen. Ferner wird in Zukunft auch auf die Einzelstoffe in diesem Abwasser zu achten sein, da bekanntlich Stoffgemische andere Wirkungen haben als die einzelnen beteiligten Stoffe. Derartige differenzierte Analysen des DEA-Abwassers stehen noch aus; sie sind aber in Zusammenarbeit mit dem DEALabor vorgesehen.

\section{ZUSAMMENFASSUNG}

1. Die DEA-Erdölraffinerie in Heide-Hemmingstedt leitet seit 1962 etwa $200 \mathrm{~m}^{3} / \mathrm{h}$ Werksabwasser nach mechanisch-biologisch-chemischer Behandlung über eine $7,5 \mathrm{~km}$ lange Rohrleitung in einen Vorlandpriel in der Nordostecke der Meldorfer Bucht (Dithmarschen, Schleswig-Holstein).

2. Der Gehalt des gereinigten Abwassers an typischen Reststoffen liegt im Durchschnitt bei etwa 5 bis $10 \mathrm{mg} / 1$ petrolätherlösliche Stoffe und etwa 1 bis $2 \mathrm{mg} / 1$ Gesamtphenole.

3. Durch gelegentliche Erhöhung desselben ist im Laufe der Zeit, wahrscheinlich unter Mitwirkung von Cyanophyceen, Diatomeen und anderen Organismen, eine Anreicherung dieser Stoffe im Schlick der beeinflußten Priele eingetreten, welche das Hundertfache und mehr des Gehalts im Abwasser ausmachen kann.

4. Dabei sind die Anteile der Ole und Fette aus pflanzlichen und tierischen Organismen in Rechnung zu setzen. Sie kommen zu dem genannten Olgehalt des Abwasserschlicks hinzu und erhöhen ihn vermutlich in beträchtlicher Weise.

5. Die Bestimmung des Phenolgehaltes im Abwasserschlick nach den üblichen Einheitsverfahren ist problematisch, da die gleichen chemischen Reaktionen auch durch andere Stoffe als Phenole hervorgerufen $\mathrm{zu}$ werden scheinen. Diese Vermutung erfordert weitere Untersuchungen.

6. Der Schlick der vom Wasser regelmäßig beeinflußten Priele und Gräben ist von anderer Konsistenz als der Schlick vergleichbarer unverschmutzter Priele, teils weicher, teils härter und meist sulfidreicher. Bei der Entstehung dieser Bodenverhältnisse spielen vermutlich besonders Cyanophyceen eine Rolle.

7. Die Biozönosen dieses Priel-, Graben- und Wattgebietes sind durch das Abwasser großenteils wesentlich beeinflußt. Cyanophyceen haben sich als gegen chemische Gifte wenig empfindliche Organismen übernormal ausgebreitet. Diatomeengesellschaften sind relativ wenig vom Abwasser verändert worden. Die Makrofauna ist im Abwassergebiet merklich verarmt. Viele Arten fehlen, nur Nereis diversicolor, ein recht euryplastischer Polychaet, hat sich an den meisten Stellen gehalten.

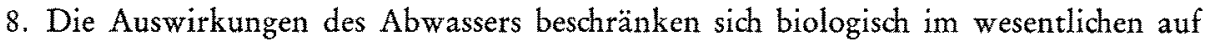
den eigentlichen Abwasserpriel und seine Nebengräben. Der mehr und auch zur Niedrigwasserzeit. Laufende weitere Kontrollen sind dennoch notwendig, da die wasserauswirkungen. Laufende weitere Kontrollen sind dennoch notwendig, da die Dauerwirkungen solchen Abwassers nicht vorauszusehen sind. 


\section{ZITIERTE LITERATUR}

Brockmann, Ch., 1950. Die Wattdiatomeen der Schleswig-Holsteinischen Westküste. Abh. senckenb. naturforsch. Ges. 478.

Hustedt, F., 1939. Die Diatomeen-Flora des Küstengebietes der Nordsee vom Dollart bis zur Elbemündung. I. Abh. naturw. Ver. Bremen 31, 572-677.

- 1955. Marine littoral diatoms of Beaufort, North Carolina. Bull. Duke Univ. mar. Stn 6.

- 1962. Die Kieselalgen. In: L. Rabenhorsts Kryptogamen-Flora von Deutschland, Osterreich und der Schweiz. Geest \& Portig, Leipzig, Bd 7, T. 3, 267.

JAсOBs, H. D., 1965. Abwasserreinigung in der Mineralölindustrie. Gas- $u$. WassFach 106 (26). Linke, O., 1939. Die Biota des Jadebusenwatts. Helgoländer wiss. Meeresunters. 1, 201-348.

WetTeramt Schleswig, 1967. Wetter in Schleswig-Holstein 1967. Wett. Schlesw.-Holst. (fortges. als:) Schnellber. dt. WettDienst Schlesw.-Holst.

\section{Diskussion im Anscbluß an den Vortrag KöNIG}

GUNKEL: Bis zu welcher Entfernung von der Einlaßstelle sind normalerweise Ölverschmutzungen nachweisbar?

KöNIG: Die grobsinnlich erfaßbaren Auswirkungen enden im allgemeinen im Hafenpriel etwa in den ersten 100 Metern beiderseits der Mündung des Abwasserpriels. Gelegentlich sind hier noch Olschlieren zu sehen. In der Besiedlung sind wieder annähernd normale Verhältnisse vorzufinden. Doch erfordert der Auswirkungsbereich des Abwassers in diesem größeren Vorfluter in verschiedener Hinsicht weitere Aufmerksamkeit, da auch mit unangenehmen Auswirkungen gerechnet werden muß.

Tomczak: Bei Sturmfluten werden die hochgelegenen Wiesen sicher überflutet. Meine Frage geht deshalb dahin, ob die Untersuchungen nicht nur in den Prielen gemacht, sondern auch auf die Wiesen ausgedehnt worden sind. Weiterhin stellt sich die Frage, ob Auswirkungen auf die Schafzucht festgestellt worden sind. Hat sich das Abwasser auch auf den Vorlandbiotop ausgewirkt, und ist das untersucht worden?

KöNıG: Eine katastrophale Einwirkung auf das Vorland erfolgte im Herbst 1962, als durch Dammbruch eines Olschlammbeckens bei der DEA viel Olschlamm in die Abwasserleitung und wegen hohen Wasserstandes auf große Flächen des Vorlandes gelangte. Ein erheblicher Schaden an der Vorlandwiese als Schafweide war die Folge. Auf diesen in solchem Umfang einmaligen Zwischenfall wird in der gedruckten Veröffentlichung näher eingegangen. Nähere biozönotische oder faunistisch-floristische Untersuchungen über Abwasserwirkungen auf das Vorland habe ich nicht anstellen können; Herr Heydemann (Zoologisches Institut der Universität Kiel) befaßt sich aber im Rahmen seiner terrestrischen Küstenforschung mit solchen Arbeiten.

HExDEMANN: In unserer Forschungsgruppe in Kiel laufen seit etwa 5 Jahren speziell in der Meldorfer Bucht, also im Untersuchungsgebiet von Herrn KöNIG, Untersuchungen über die terrestrischen Arthropoden des gesamten bewachsenen Vorlandes. Diese experimentell-ökologischen Freilanduntersuchungen geschehen mit automatischen, uberflutungssicheren Fallen, deren Ergebnisse sich gut quantitativ auswerten lassen. Es ergab sich anläßlich einer Verölung des Salzwiesengebiets im Jahre 1965 stellenweise ein Rückgang von Bodenarthropoden aus der Gruppe der Amphipoden (Orchestia) und der Coleoptera (Carabidae, Staphylinidae) auf etwa 50 bis $25 \%$ des Normalwertes. Unsere Arbeiten konzentrieren sich momentan auf experimentellökologische Untersuchungen im Labor in bezug auf die Auswirkungen der Abwässer dieser Olindustrie auf typische Arthropoden des beweideten Vorlandes. Erst nach Vorliegen dieser Ergebnisse lassen sich genaue, artspezifische Aussagen zu diesem Problem machen. 